\title{
Experimental determination of the overturning moment and net lateral force generated by a novel VAWT - Experiment design under load uncertainty
}

\author{
A._Kolios ${ }^{1, \mathrm{a}}$, A. Chahardehi ${ }^{1}$, F. Brennan ${ }^{1}$ \\ ${ }^{1}$ Department of Offshore, Process and Energy Engineering, Cranfield University,
} Bedfordshire MK43 0AL, United Kingdom.

aa.kolios@cranfield.ac.uk

\begin{abstract}
Recent developments in harnessing wind energy propose new, radically different designs to alleviate some of the difficulties associated with conventional wind turbines. New designs however require testing for a variety of reasons ranging from gaining confidence in the analytical models used in the design and development through to satisfaction of certification requirements. Medium-scale prototype testing of large scale concepts, where parameters such as the response of the structure and the loading conditions are often highly uncertain demand special consideration. This paper presents the design of a special test rig and calculation methodology for the experimental determination of the overturning moment and net force generated by the NOVA Vertical Axis Wind turbine (VAWT) using a field experiment set up. The design of the experimental model involves dealing with modelling uncertainties since loads in operation and therefore the response of the structure are largely unknown before testing has been carried out. The variability in the wind speed and direction also need to be accommodated for.
\end{abstract}

Keywords: Experiment under Uncertainty, Wind Turbine, Vertical Axis Wind Turbine, Overturning Moment and Force Determination, NOVA Wind Turbine 


\section{INTRODUCTION}

Deployment of wind farms during the last decade has highlighted some difficulties associated with conventional turbines. Conventional Horizontal Axis Wind Turbines (HAWTs) place their rotors and drive-train at the top of very tall towers, making installation difficult and limiting their size; maintenance is also difficult because of the sheer height of all the moving parts. Vertical Axis Wind Turbines can overcome the disadvantages mentioned above by locating their main components at the base of the installation, providing easy access and a lower value of overturning moment, and are less sensitive to wind direction. Riegler ${ }^{1}$ and Ericsson et al ${ }^{2}$ provide some interesting analytical discussions for these different design philosophies. On the other hand, in the conventional VAWTs based on the Darriues concept a large overturning moment exists and is transferred to the tower which can lead to severe fatigue design provisions. The current novel design is deemed to have overcome this problem by reducing the overturning moment. Having a large tower further exacerbates the situation by adding drag forces which act as overturning moments at the root of the tower.

In this paper, the experimental determination of the generated overturning moment and net lateral force for a novel VAWT is considered through a prototype field test. In this new turbine design the over-turning moment is greatly reduced, increasing stability and reducing the size of support structure required. The NOVA $^{3}$ turbine is a vertical axis wind turbine where the philosophy of the design is to create a low value of the overturning moment with the aid of the sails attached to the V-shaped rotor. Figure 1 shows the concept of the NOVA aerogenerator ${ }^{4}$. In this design, the wind forces on the sails will result in an overturning moment which counteracts the overturning moment generated by the action of the wind on the V-shaped arms, and therefore the effect of the sails is a net reduction in the algebraic value of the overturning moments.

The effect of the sails in reducing the overturning moment can be described as follows. The effect of wind force on the arm is translated as a net overturning moment and a shear force at the base of the arm. However, addition of a sail will create a new net force acting on the sail, which when translated to the hub, will act as a net shear force, and since the line of action of the resultant force acting on the sail is not parallel to the arm, this results in a net overturning moment. The relative direction of this component of 
overturning moment about the hub depends on the angle at the intersection between the sail and the arm, as shown in Figure 2. As can be seen from the figure, by changing the direction of the wind, the directions of the two overturning moments from the arm and the sail change and therefore this counteracting effect still holds.

\section{EXPERIMENTAL TESTING}

Experimental testing is one of the most important tools in the development process of a new design, since it can provide real data that will advance the design or, when executed in a later phase, can reveal potential issues that need more consideration. This is applicable in the case of any new technology ${ }^{5}$, or in cases where proven technologies are employed in new environments, and stands as a basic requirement towards certification. Beheshti ${ }^{6}$ gives an analytical procedure of a comprehensive design process that can also be applicable to the design of experiments. In the $\mathrm{DnV}$ classification note $30.6^{7}$, a classification of uncertainties is presented distinguishing physical, measurement, statistical and model uncertainties. In the present paper, the effect of model uncertainty will be investigated as a source of uncertainty based on imperfections and idealisations made on the design of the physical model due to the inability to predict the actual loading conditions.

In field testing, the effects of environmental loads are significant but difficult to predict. On the other hand, and this is particularly important in the measurement of forces, the response of the structure should be of a certain magnitude within specific limits in order that these can be reliably measured. In this case, where the wind is the basic 'input' of the system, the effect of its uncertain magnitude can directly influence the reliability of the testing results as well as the duration and total cost of the experiment.

The mechanism devised and the procedure that was followed for the NOVA wind turbine testing are applicable to any wind turbine, or indeed in any similar experiment where the magnitude of the measured forces is not known beforehand. This design and the associated procedure are presented in this paper. 


\section{PROBLEM DEFINITION}

One of the key advantages of the vertical axis wind turbine configurations is the low overturning moment when compared to conventional horizontal axis wind turbine designs. The magnitude of the static overturning moment with the rotor parked (stationary rotor) in extreme gust cases is however, a critical design parameter for the overall design concept. Static overturning moment data is hence a valuable measurement for the design and optimization studies and subsequent certification.

In the experiment outlined herein, in order to measure the lateral force and overturning moment exerted by the stationary rotor, the rotor was mounted on a platform on top of a slender tubular structure. The additional height provided by the tower enabled the rotor to experience clean, undisturbed wind, with no buildings or other obstructions to generate turbulence. In addition to this, as described in the next section, the tubular tower allowed attachment of two sets of strain gauges at different elevations on the tower in order to obtain the data required to calculate the overturning moment and the horizontal force. The tower along with the rotor is shown in Figure 3. Measurements are taken for stationary rotor with different orientations. The final results are sorted in terms of the relative angle between the wind and the rotor.

\subsection{CALCULATION PROCEDURE}

By experimentally obtaining the bending moments at two different heights on the tower, the overturning moment and the net horizontal force can be evaluated. These bending moments will be obtained from the straingauges mounted on the tower. Considering known values for this bending moment at the locations A and B as shown schematically in Figure 4, the rotor overturning moment and net horizontal force will be calculated as follows, the effect of wind drag on the tower can also be accounted for as a linear distribution of lateral force along the length of the tower:

$$
\begin{gathered}
M_{@ A}=M+F \times A C \\
M_{@ B}=M+F \times B C
\end{gathered}
$$




$$
\begin{gathered}
\rightarrow F=\frac{M_{@ A}-M_{@ B}}{A B} \\
\rightarrow M=M_{@ B} \times \frac{A C}{A B}-M_{@ A} \times \frac{B C}{A B}
\end{gathered}
$$

In order to determine the bending moment values at heights A and B (see Figure 4), a set of three strain gauge rosettes are attached around the periphery of the tower at each of the two heights. The strain readings from these measurements can be transformed to stresses according to the following fundamental equations:

$$
\begin{gathered}
\varepsilon_{0^{\circ}}=\varepsilon_{x} \varepsilon_{90^{\circ}}=\varepsilon_{y} \\
\varepsilon_{\theta}=\varepsilon_{x} \cdot \cos ^{2} \theta+\varepsilon_{y} \cdot \sin ^{2} \theta+\gamma_{x y} \cdot \sin \theta \cdot \cos \theta \\
\varepsilon_{45^{\circ}}=\frac{1}{2} \cdot \varepsilon_{0^{\circ}}+\frac{1}{2} \cdot \varepsilon_{90^{\circ}}+\frac{1}{2} \cdot \gamma_{x y} \rightarrow \gamma_{x y}=2 \cdot \varepsilon_{45^{\circ}}-\left(\varepsilon_{0^{\circ}}+\varepsilon_{90^{\circ}}\right)
\end{gathered}
$$

The superficial stress state at each of the three points on the surfaces of the strain gauged cylinders is then given as:

$$
\begin{gathered}
\sigma_{x}=\frac{E \cdot\left(\varepsilon_{x}+v \cdot \varepsilon_{y}\right)}{1-v^{2}} \\
\sigma_{y}=\frac{E \cdot\left(v \cdot \varepsilon_{x}+\varepsilon_{y}\right)}{1-v^{2}} \\
\tau_{x y}=\frac{E \cdot \gamma_{x y}}{2 \cdot(1+v)}
\end{gathered}
$$

From which the bending moment values $\boldsymbol{M}_{@ \boldsymbol{A}}$ and $\boldsymbol{M}_{® \boldsymbol{B}}$ can be determined.

\subsection{GENERAL CONSIDERATIONS}

In order to obtain adequate data for the procedure mentioned above, two points at different elevations should be considered on a tubular tower that will stand as a support structure of the Vertical Axis Wind 
Turbine (Figure 4). The tower should be designed to resist the dead load of the turbine as well as its operational loads. This refers to a torsion load due to the rotation of the rotor, an overturning moment and a thrust force due to the drag of the tower and the rotor against the wind. The response of this prototype structure to wind is considered initially to be unknown. However, strain gauges are most accurate within a certain, relatively narrow range of applied stresses, and overdesign of the structure would result in very low and therefore unreliable and inaccurate measurements. Therefore the optimum 'window' of stiffness has to be evaluated in order to obtain reliable stress values in a safe manner.

\subsection{DETAILS OF THE MEASUREMENT MECHANISM}

The issue of the unknown response of the structure, considering also the unknown wind conditions at the design phase, is dealt with by employing two concentric tubes that can be operated individually, giving strain measurements within the acceptable accuracy range of the strain gauges; the mechanism is shown in Figure 5. The dimensions of the tubes selected are based on a design assuming normal wind conditions for the proposed testing site (RAF Bentwater, Suffolk, UK) that where based on existing data records ${ }^{8}$ for a normal wind velocity of $12 \mathrm{~m} / \mathrm{sec}$ and for the response of the structure, a conservative drag calculation was employed. Since the response of the structure is unknown, the smaller diameter tube will be operated in case of a wind or response of the structure lower than expected so that the strains are high enough in magnitude to be accurately detected by the strain gauges.

The details in the two elevations have been sized individually to account for the different loads they have to resist. Obviously the lower detail consists of tubes with greater thicknesses as they have to resist the dead load of the intermediate part of the tower and, more importantly, the magnified overturning moment due to distance of the applied forces.

In normal conditions, initial measurements are taken with the large cylinder engaged. At this layout, the inner-smaller diameter cylinder will remain loose inside the large one, carrying no load. If those measurements are found to be small, the smaller tube will be put into operation by tightening the inner set of bolts through the clearance between the bottom slip-on and blind flange as shown in Figures 5 and 6 . In this case, bolts of higher grade, 12.9, were selected with nuts welded below on the smaller flange, in order 
to axially carry the distributed weight of the structure and therefore be able lift and engage the smaller cylinder and allow the larger pipe to become load-free. When the larger tube is in operation, this clearance is kept fixed, through the use of adequate size collars.

For safety reasons, in conditions of extreme wind speed, guyed wires are connected from eyebolts on the top flange reaching to fixed concrete ballast at ground level. For the weaker details, $500 \mathrm{~mm}$ collars stiffen the smaller cross section and studs from the upper slip-on flange through the collars and the lower set of flanges are used. Figure 6 shows where the bolts are tightened in order to switch between the load-carrying tubes.

It was found during the experiment that switching from the outer cylinder to the inner one so that all the loads are carried by this cylinder was easily achievable using conventional tools. However, it must be noted here that in order to obtain accurate and meaningful measurements, one must ensure that the disengaged tube does not carry any of the external load. This can be achieved by ensuring that the strain readings from the gauges on this cylinder indicate 'zero' values as calibrated prior to the experiment.

\subsection{INSTRUMENTATION}

The tube gauges were applied before the flanges were welded therefore in order to resist heat, the gauges were bonded with M Bond 600 (care was taken not to damage the cable during the welding with the use if wetted cloths etc). Products used in the installations are BSSM ${ }^{9}$ approved and supplied by Vishay Measurements Group ${ }^{\circledR}$.

Three rosettes were installed around the periphery of the cylinders at the two elevations. In total, there were two pairs of concentric cylinders each containing three rosette strain-gauges, giving a total of 36 strain readings. However, at every given instance, only one of each pair of cylinders was carrying the load and therefore a total of 18 readings were used for the final stress analysis of the tower. On each cylinder, the three rosettes were attached around the circumference spaced at $120^{\circ}$ as shown in Figure 7.

The installed strain-gauge system was verified and calibrated prior to measurement of overturning moment. This was achieved by application of a known force to one of the guyed cables while all other three cables 
were fully slack. Calibration of the rosette strain-gauge on the inner and outer cylinders was achieved by application of an incrementally increasing force on one of the guyed wires and then decreasing the force at similar increments and registering the strain readings. This calibration was applied to the strain-gauge readings during the post-processing of the results.

The rosette arrangements on the tower make it possible to measure torque generated from a stationary rotor for different wind speeds. However this can also be achieved very easily by the conventional use of torquemeters. The torque measurements give valuable information about the power performance of the rotating turbine. In the stationary case, however, the toque value is not an important parameter in the design of the current experiment.

\section{TEST PROCEDURE}

Prior and during the experiment, the strain gauges were calibrated in order to ensure accurate strain readings within the range expected strains. During the experiments, two tests were conducted simultaneously: wind data mesaurement and strain measurements. The wind speed and direction was measured throughout the testing period by use of an anemometer mounted on a scissorlift raised to the mid height of the rotor. The strain measurements (18 channels) were collected and stored using the time signature to allow matching of the wind and strain data. The strain measuremens were taken for different orientations of the rotor relative to the magnetic north, the exposure time being 15 minutes for each test. Then the combined wind-strian data were arranged for different relative angles between the wind and the plane of the rotor. The strain results were then transformed into stresses, and subsequently into bending moments, which then by the application of the procedure outlined in 3.1, were converted into force and moment values for different wind velocity magnitudes.

The way the data are sorted is as follows. For each orientation of the rotor the exposure time is 15 minutes. The data from this duration are then sorted in bins of relative wind direction. Within each band of direction, the data are further 'binned' with respect to the magnitude of the wind speed. The bandwidth of these wind speed bins are chosen in a way to contain enough data points in order to obtain a meaningful 
mean value for each bin. The wind speed statics are also computed and the distribution of direction and velocity magnitude have been obtained as part of the test.

\section{RESULTS}

The objective of the experiments was to assess the response of the stationary rotor to different wind conditions. From the experiments, values of the overturning moment and the lateral force were derived for different wind speeds under different wind directions. Some of the trends obtained from these results are shown in the Figures 9 and 10. These figures show the normalised values of the lateral force (Figure 9) and the overturning moment (Figure 10) for two different relative wind directions, namely $10^{\circ}$ and $30^{\circ}$. The trends observed in the experimental measurements of forces and moments are in general agreement with the analytical predictions that are based on the aerofoil theory and the forces and moments acting on stationary bodies due to the action of the wind. This simple model lacks turbulence effects and is therefore only an indication of the trends of change of forces and moments as a function of the wind speed and orientation.

The values of the force and overturning moment have been normalised by dividing them by the values for a wind speed of $3.5 \mathrm{~m} / \mathrm{s}$. This would make comparison of the trends easier for different wind directions.

\section{SUMMARY AND CONCLUSIONS}

In this paper a novel mechanism was introduced which was used to account for the inherent uncertainty in the loadings on the measurements sensors. In HAWTs, traditionally the effect of wind loading on the rotor is measured by straingauge measurements at the blade root. However, this does not allow for separating the horizontal force and the net overturning moment in the turbine. Measurement of the net horizontal force in HAWTs is also a complicated problem but the effect of this force can be measured with relative ease by straingaguing the tower. It is traditionally assumed that the net overturning moment at the hub level of a HAWT is zero or negligible. 
The double concentric tubes, along with the mechanism shown in Figue 5, allow for accurate measurement of the reponse of the wind turbine in a range of wind speeds; some of these measured results are shown in this paper. Experience obtained from this experiment, shows that environmental loading is a considerable unknown that can affect the efficiency of the results. Design variations, such as use of a mechanism as described above, can expand significantly the range of effective wind velocities between the minimum allowed from the strain measurements and the maximum that might compromise the integrity of the test rig. The stain-gauge double tube system also allows for some a priori uncertainty in the response of the rotor under the action of a known wind speed, therefore showing a large scope for similar experimatation. In the case of the NOVA test case, this range, was doubled allowing reliable measurements in an instant with low wind velocities, avoiding undesirable delay of data collection.

Efficiency of the current method can be highlighted when compared to a method where force transducers are used instead of strain gauge rosettes. The decoupling of the force and overturning moment is only possible by taking measurements, by strain gauges or force transducers, at two different elevations. Use of transducers is conditional to being able to embed them in the tower in a way to put them in the load path of the structure. This means a more complicated design for the tower. In order to accommodate for uncertainty in the measurement, the magnifying effect of a tower with a smaller cross section cannot be utilized when using force transducers.

The current technique is quite versatile since fewer number of straingauges give the full force/moment data about the turbine. A larger number of expensive transducers should be employed to derive the same data.

\section{ACKNOWLEDGEMENTS}

This study formed part of a project funded by the Energy Technologies Institute (ETI), a unique partnership between global industries and the UK Government. The six current private sector partners are BP, Caterpillar, EDF Energy, E.ON, Rolls-Royce and Shell. The ETI's public funds are received from the Department for Innovation, Universities and Skills (DIUS) through the Technology Strategy Board (TSB) and the Engineering and Physical Sciences Research Council (EPSRC) with additional funding from the 
Department for Transport.

References

1. Riegler, Hannes. HAWT versus VAWT: Small VAWTs find a clear niche . Refocus. 2003, Vol. 4, 4.

2. Sandra Eriksson, Hans Bernhoffa and Mats Leijona. Evaluation of different turbine concepts for wind power, Renewable and Sustainable Energy Reviews. 2008, Vol. 12, 5.

3. The NOVA Project, www.nova-project.co.uk

4. Sharpe, D.J., WO/2006/054091, Vertical Axis Wind Turbine Apparatus, 2006.

5. Veritas, Det Norske. DNV-RP-A203: Qualification proceedures for a new technology. Norway: Det Norske Veritas, 2001.

6. Beheshti, Reza. Design decisions and uncertainty. Delft University of Technology, The Netherlands : Butterworth-Heinemann Ltd, 1993.

7. Veritas, Det Norske. 30.6 Classification Note: Structural Reliability Analysis of Marine Structures. Norway: Det Norske Veritas, 1992.

8. http://www.tutiempo.net/en/Climate/BENTWATERS_RAF/35961.htm [Accessed online in October 2009]

9. British Society for Strain Measurement, http://www.bssm.org/ 
Figure 1: The NOVA turbine concept

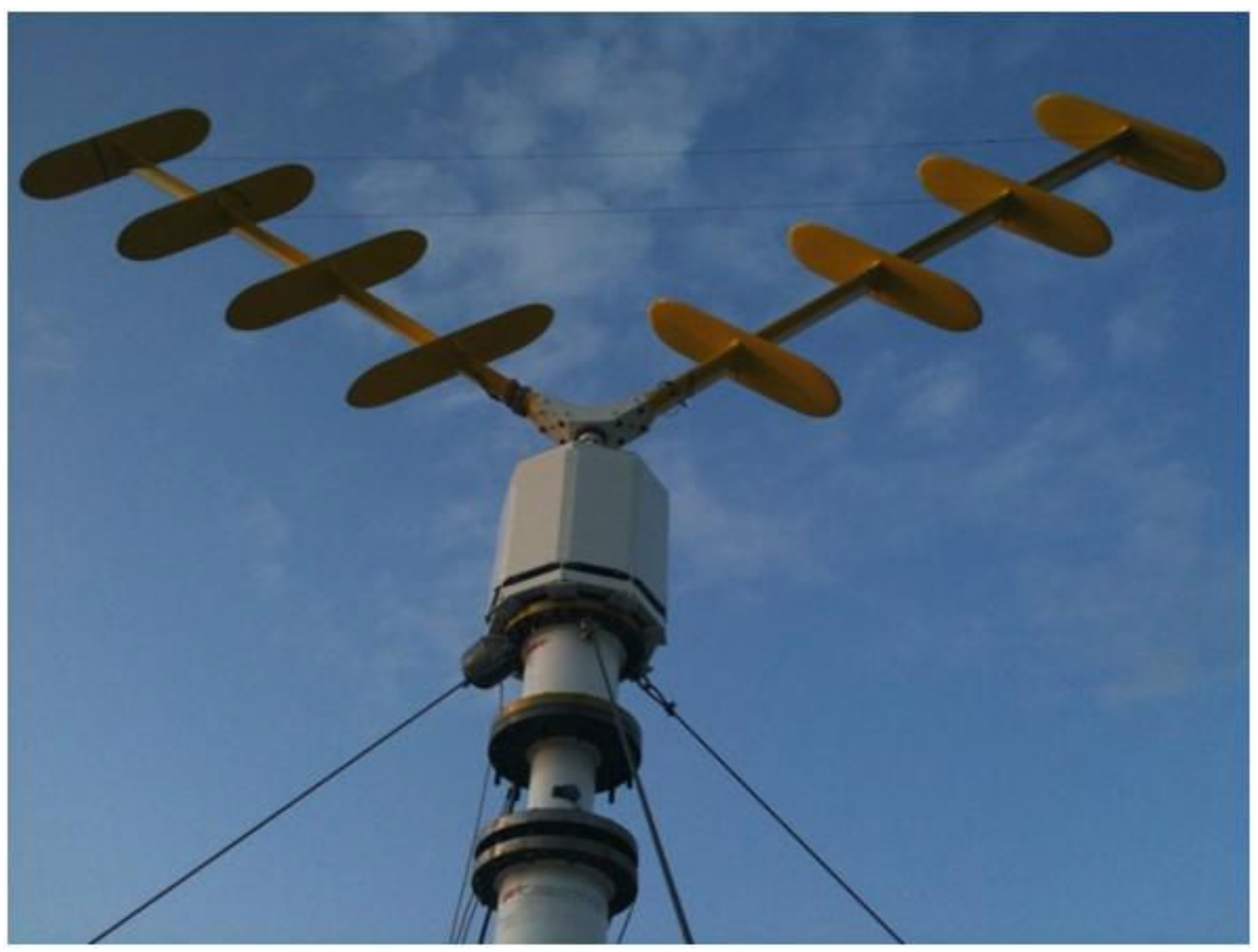

12 
Figure 2: Wind forces acting on the rotor

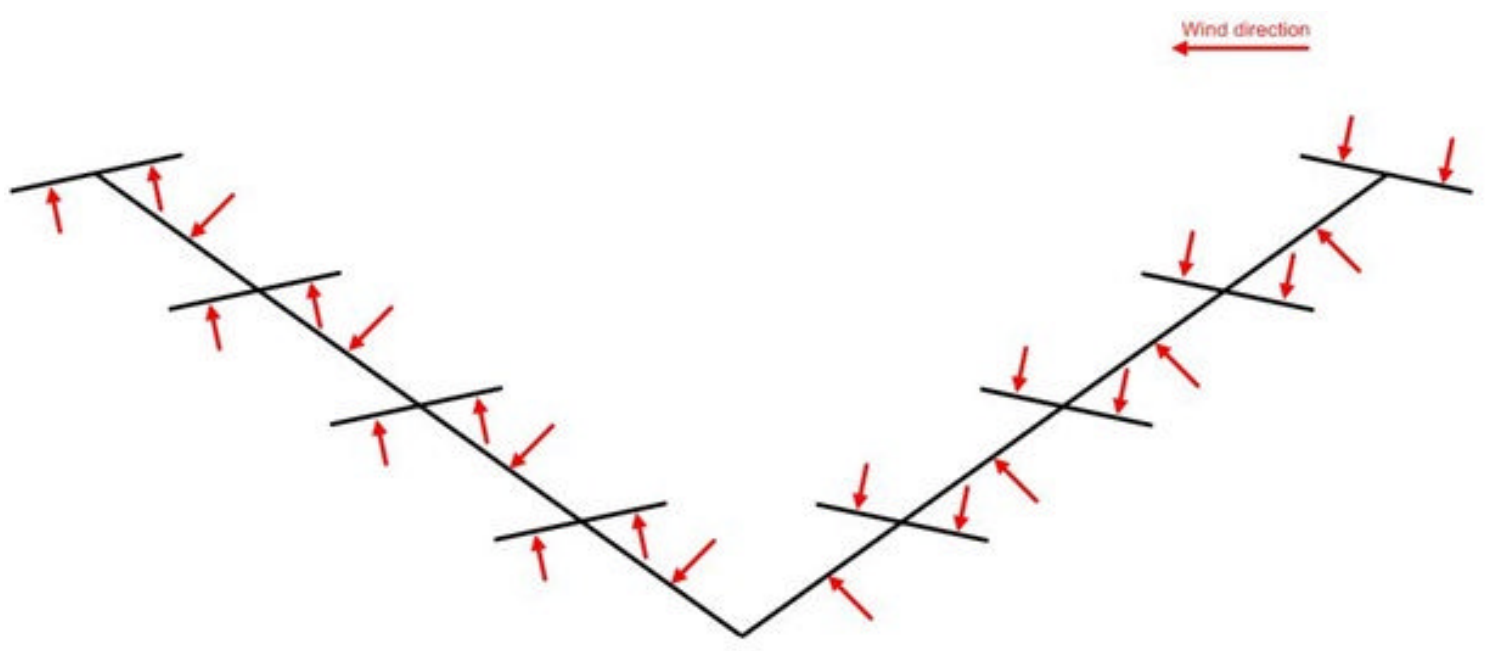

13 
Figure 3: The test tower set up

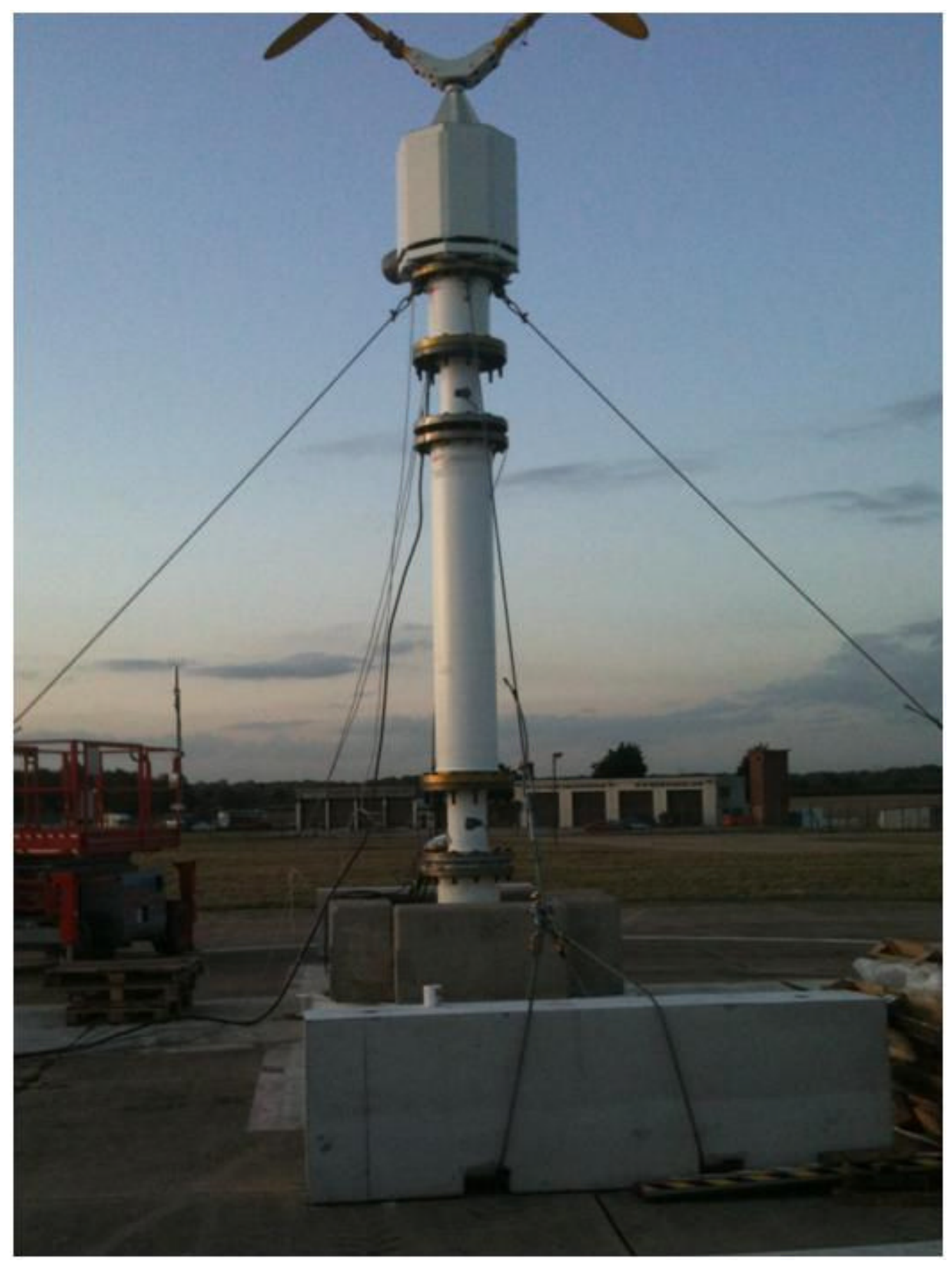


Figure 4: Test tower and schematic representation of the loads from the turbine on the tower

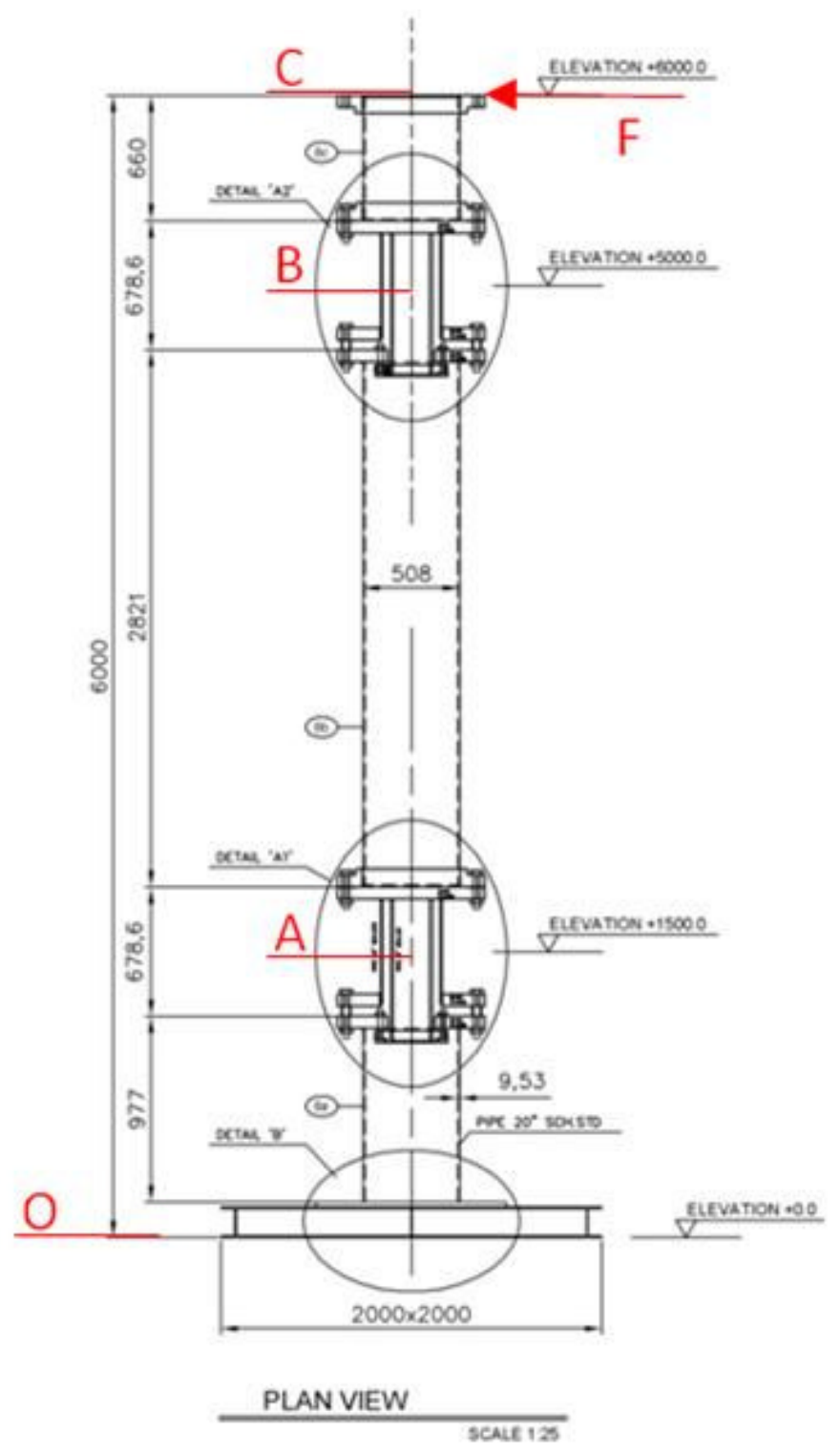


Figure 5: Configuration of the mesurement mechanism
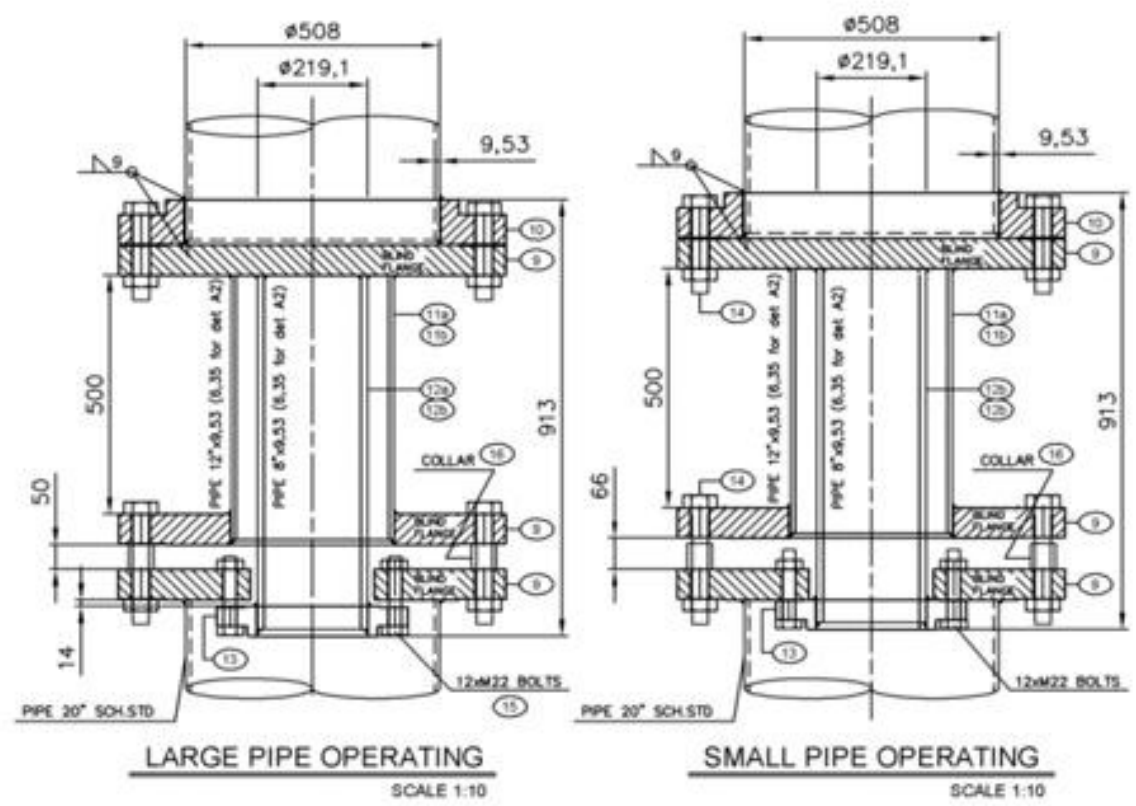
Figure 6: Tightening the bolts in order to switch between tubes

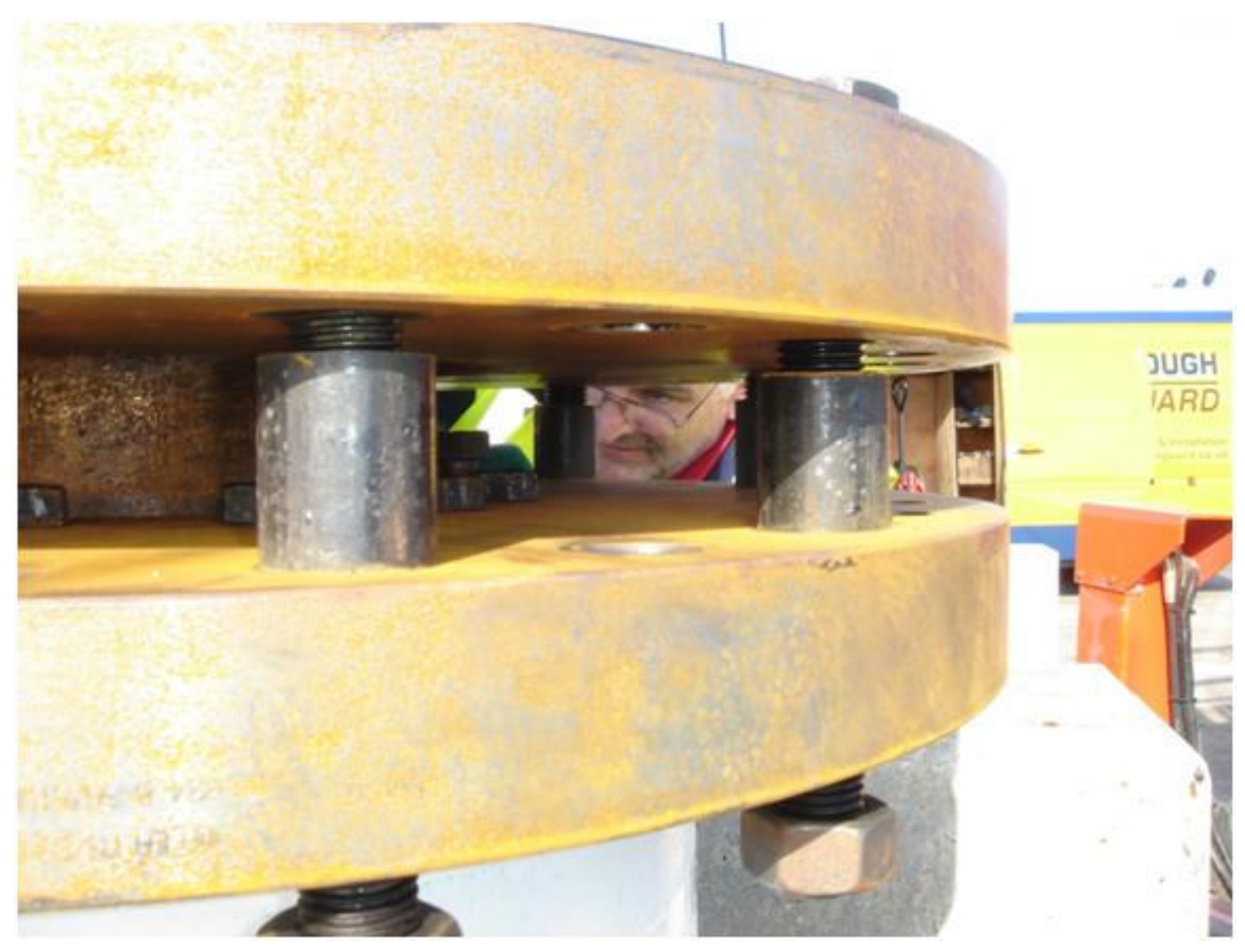


Figure 7: Cross section of each cylinder showing the orientation of the rosette around the tube

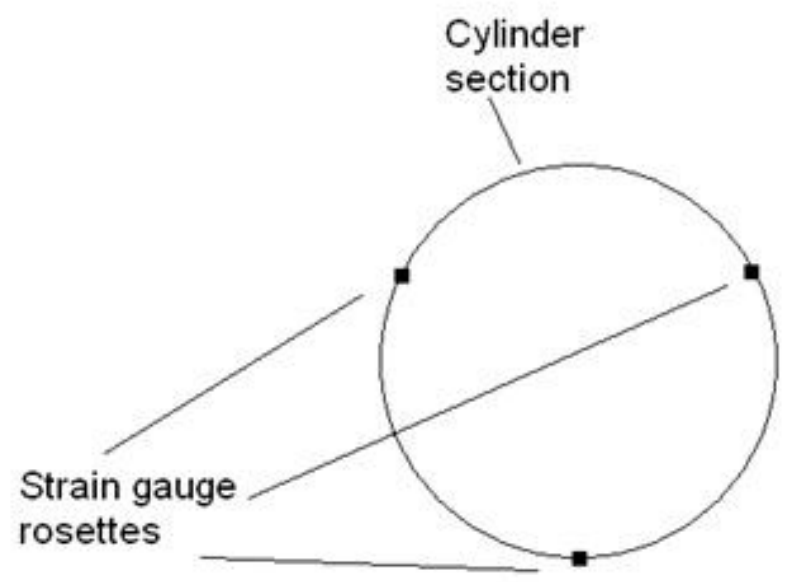


Figure 8: Rotor orientation with respect to wind direction

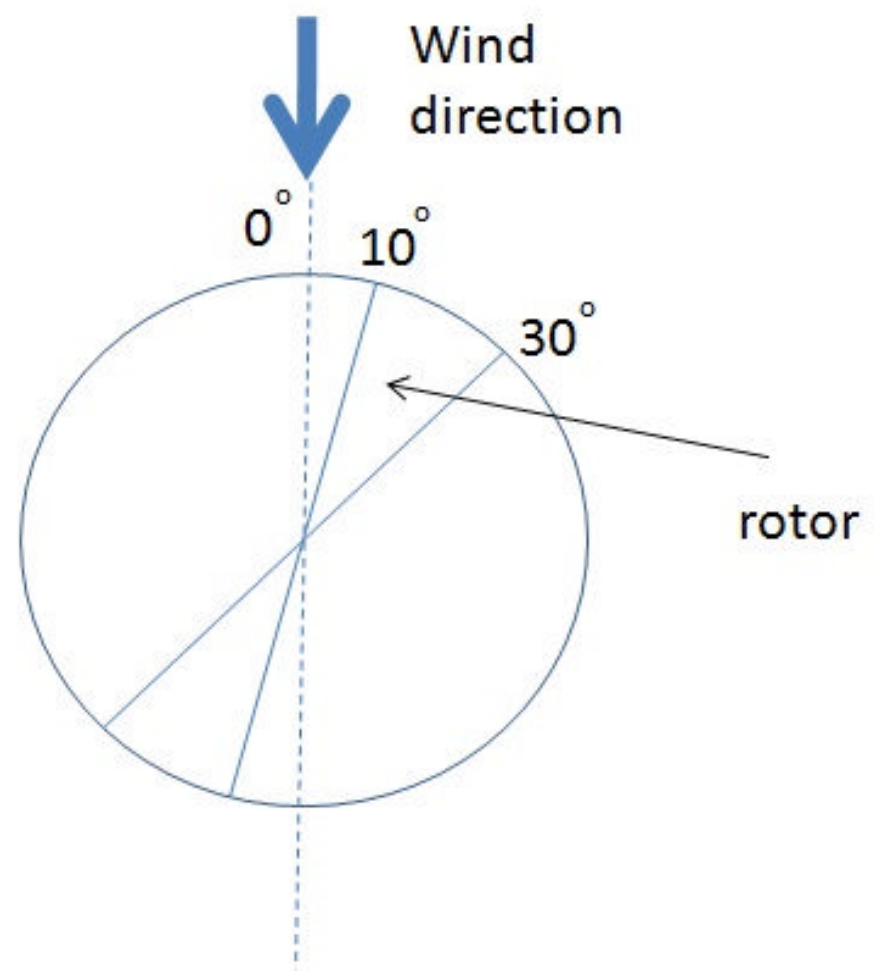


Figure 9: Normalised lateral forces for different wind speeds at two different wind angles

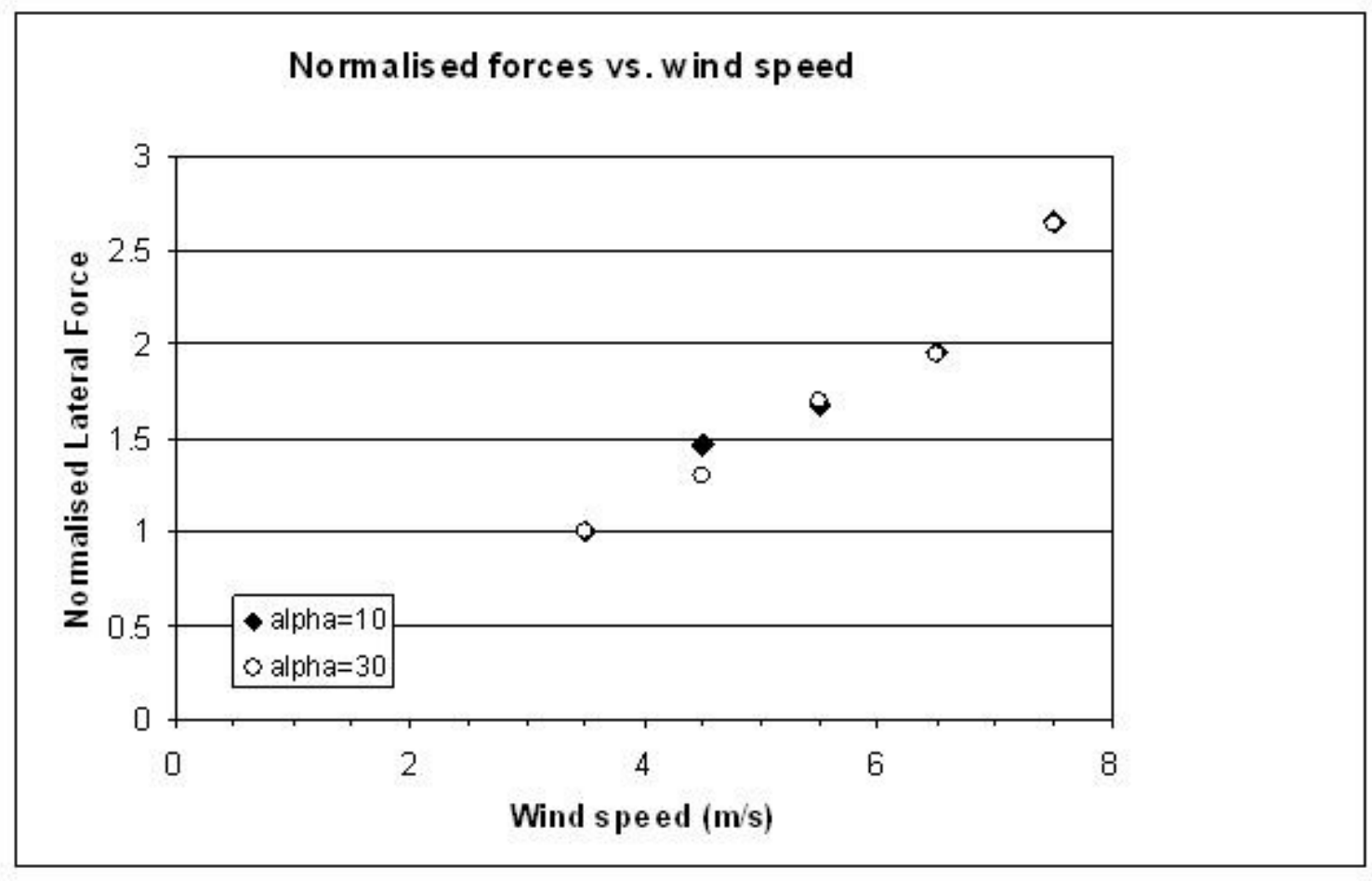


Figure 10: Normalised overturning moments for different wind speeds at two different wind angles

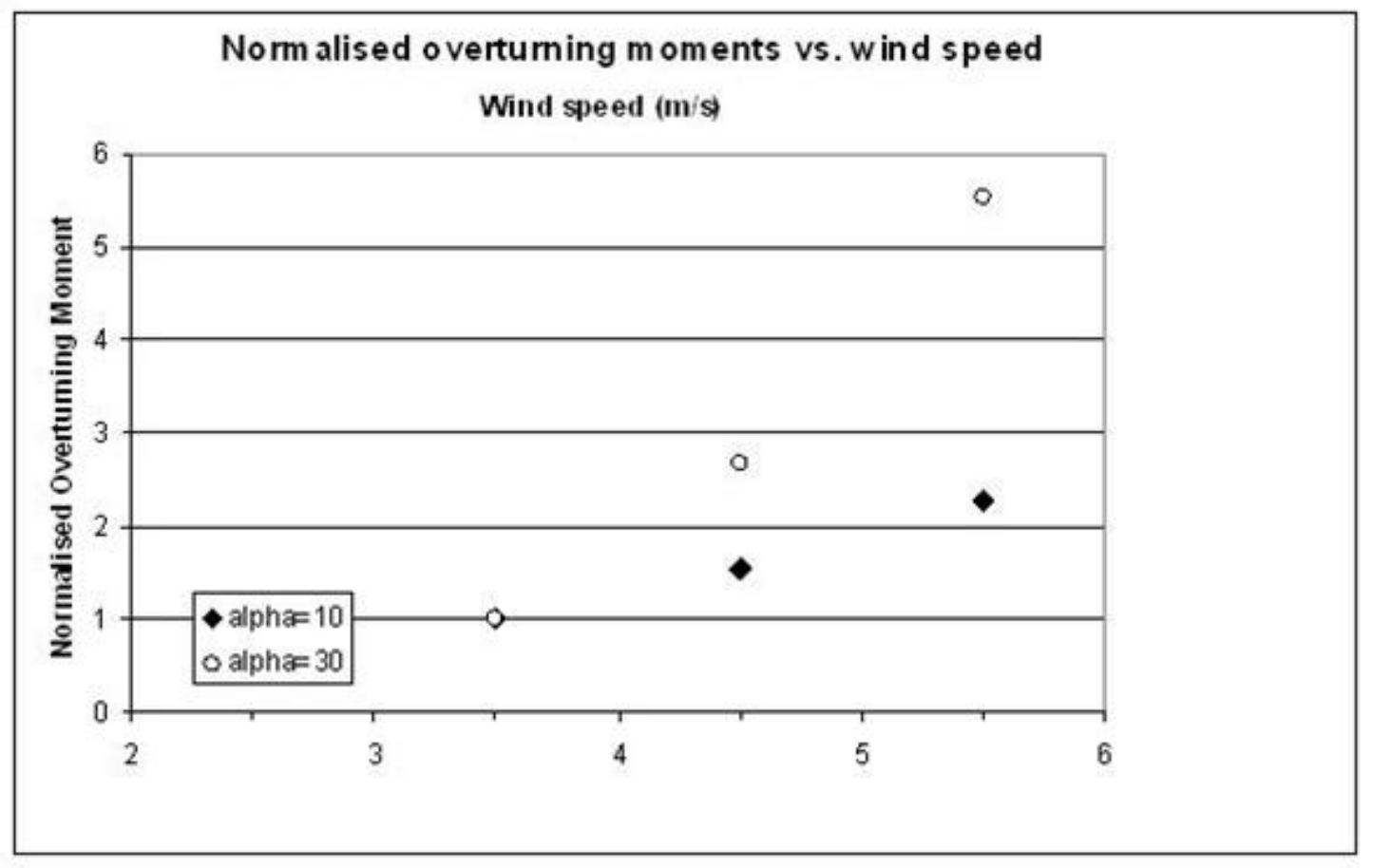

